\title{
Experimental Investigation of Dust Density Waves and Plasma Glow
}

\author{
Oliver Arp, David Caliebe, Kristoffer Ole Menzel, Alexander Piel, and John A. Goree, Member, IEEE
}

\begin{abstract}
Dust density waves (DDWs) are compressional modes that are often excited by subsonic ion flows in dusty plasmas. Previous experiments relying on imaging of only the dust revealed that they can propagate parallel to the ion flow direction or at an oblique angle. An experiment was performed using microgravity conditions on parabolic flights with video imaging of both the dust and the plasma glow. Glow arises from electron-impact excitation of neutral gas atoms, and it serves as a signature of energetic electrons. Averaging over time, it was found that the presence of dust enhances the glow brightness everywhere in the plasma. Resolving the time variation, a spontaneously excited DDW was observed at 3.9 Hz. It was characterized not only by a compression of the dust number density but also by a modulation of the glow intensity. The correlation between the wave and the glow is analyzed by Fourier methods. We found an unexpected phase relation between the plasma glow and the DDW of $118^{\circ}$. A glow maximum is followed by a dust density maximum.
\end{abstract}

Index Terms-Dusty plasma, microgravity, plasma glow, selfexcited dust density waves (DDWs).

\section{INTRODUCTION}

D UST density waves (DDWs) are a highly discussed topic in dusty plasmas. This instability has been observed in many different types of discharge, e.g., [1]-[4], and usually spontaneously emerges at low gas pressures and high dust densities. The fundamental mechanism that drives the DDWs is a Buneman-type instability of charged massive dust grains, which interact with streaming ions and are shielded by thermal electrons [5]. Early theories dealing with these acoustic-like waves in dusty plasmas were purely 1-D and allowed only wave propagation parallel to the ion flow [6]. In this earlier literature, the mode was often called the dust acoustic wave. However, recent experiments have shown that much more complicated situations can occur, where the propagation direction of the waves is not strictly parallel to the local ion flow [7], [8]. The authors also presented a model [4], which explains the direction of these obliquely propagating DDWs and its dependence on the ion local flow velocity. In addition to all experimental and

Manuscript received June 29, 2009. First published November 24, 2009; current version published April 9, 2010. This work was supported in part by the German Aerospace Agency (DLR) under Contract 50WM0739 and in part by the European Space Agency.

O. Arp, D. Caliebe, K. O. Menzel, and A. Piel are with the Institut für Experimentelle und Angewandte Physik, Christian-Albrechts-Universität zu Kiel, 24098 Kiel, Germany (e-mail: arp@physik.uni-kiel.de; caliebe@physik.unikiel.de; menzel@physik.uni-kiel.de; piel@physik.uni-kiel.de).

J. A. Goree is with the Department of Physics and Astronomy, The University of Iowa, Iowa City, IA 52242 USA (e-mail: john-goree@uiowa.edu).

Color versions of one or more of the figures in this paper are available online at http://ieeexplore.ieee.org.

Digital Object Identifier 10.1109/TPS.2009.2034312 theoretical work, many questions with regard to the nature of DDWs are still unanswered, including the processes that lead to saturation of the wave amplitude and the strongly nonsinusoidal character of the wave.

In this paper, we report recent experiments that have been performed under microgravity conditions on parabolic flights, in which we simultaneously imaged the dust and the plasma glow while observing self-excited DDWs. The plasma glow is a consequence of impact excitation of neutral gas atoms, which are hit by fast electrons that gained their energy from electric fields inside the plasma. In this sense, the plasma glow can be regarded as a signature of both energetic electrons and electric fields. While our glow imaging method offers useful information, it does not completely characterize the electron density and temperature, or even distinguish between the effects of higher density versus higher temperature, as has been attempted using line-ratio methods [9], [10].

\section{EXPERIMENTAL SETUP AND DATA ANALYsis}

All experiments presented here were performed on parabolic flights under microgravity conditions in the IMPF-K2 chamber, which is a modification of the device described in [4]. A schematic vertical section of the reactor is shown in Fig. 1(a). The symmetric parallel-plate discharge has two circular disc electrodes of $8-\mathrm{cm}$ diameter and a discharge gap of $3 \mathrm{~cm}$. Both electrodes are powered in push-pull mode via a matching network at $f=13.56 \mathrm{MHz}$. The radio-frequency voltage was $55 \mathrm{~V}$ peak-to-peak at the electrodes. We suppressed the selfbias by means of a shunt resistor. The discharge was operated in argon at a pressure of $20 \mathrm{~Pa}$. Spherical monodisperse melamine formaldehyde particles of 3.4- $\mu \mathrm{m}$ radius were injected into the plasma, yielding a particle density $n_{d} \approx 5 \times 10^{10} \mathrm{~m}^{-3}$.

For the simultaneous observation of the dust and the plasma glow, we used a system of two synchronized charge-coupled device video cameras (PAL, interlaced) with a resolution of $768 \times 576$ pixels and a frame rate of 50 fields/s [see Fig. 1(b)]. Both cameras observe the same field of view (FOV) via a beam splitter cube. The camera for the dust observation ("dust cam") is equipped with a narrow-bandwidth filter of 10-nm half-width, which is transparent for the light of the illumination laser sheet $(660 \mathrm{~nm})$, but blocks the plasma glow. It observes at right angle the scattered light of the dust particles inside the thin laser sheet of 1-mm width. The second camera ("glow cam") is used to observe the light emission of the plasma. To prevent any distortion by the illuminated dust particles, a short-pass filter is used, which is transparent only for wavelengths shorter than $600 \mathrm{~nm}$ and therefore blocks the light of the illumination laser. 


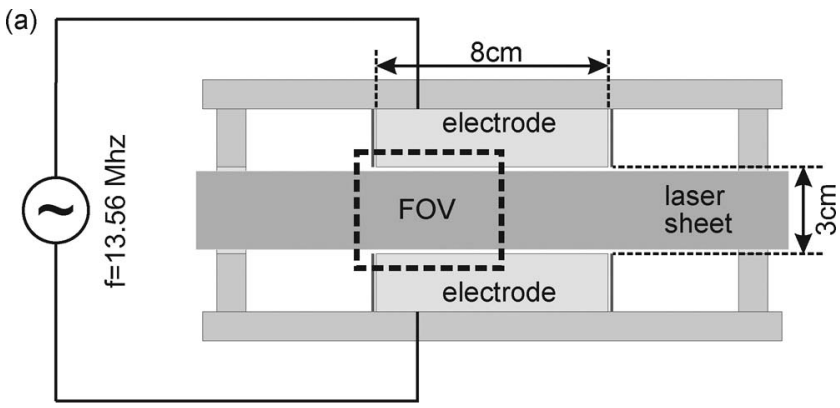

(b)

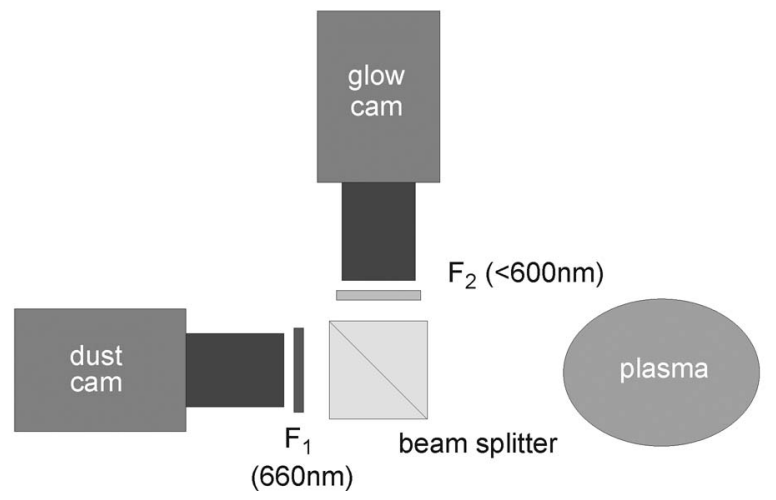

Fig. 1. (a) Vertical section of the IMPF-K2 chamber. The cameras' FOV is marked by a dashed rectangle. (b) Camera system for simultaneous observation of the dust and the plasma glow in the same FOV.

The digitally recorded video data were processed in the following way: In a first step, we use an affine transform to match the fields of view of both cameras to correct a nonperfect alignment due to mechanical tolerances. The resulting error is significantly below one pixel. Second, the video frames were deinterlaced, i.e., we separated the odd and even fields and used linear interpolation to reconstruct the missing lines. Third, the resulting image sequence was blurred by means of a Gaussian filter to remove noise and, in the case of the dust video, the granularity of the individual particles. The intensity of the blurred dust video is used as a measure for the local dust density. Fourth, for each frame of the dust and glow videos, we calculate the fluctuations in the intensity by subtracting, for each pixel, a time-average value of the same intensity. Finally, the fluctuation time series at each pixel coordinate is Fourier transformed. As described in [4], we calculate the phase from the argument of the complex Fourier coefficient corresponding to the dominant fluctuation frequency. Performing this procedure for each pixel coordinate yields a phase map.

\section{RESULtS}

A snapshot from the dust video is shown in Fig. 2(a). The two electrodes are located at the top and the bottom of the image. The image of the dust cloud is partly distorted by bright reflections of the illumination laser in the background on the chamber wall. One can clearly see self-excited DDWs, which propagate from the edge of the dust-free "void" radially outward. The wavelength of the DDW slightly varies with location inside the dust cloud. Typical values of the wavelength are $\lambda_{\mathrm{DDW}}=2-5 \mathrm{~mm}$.
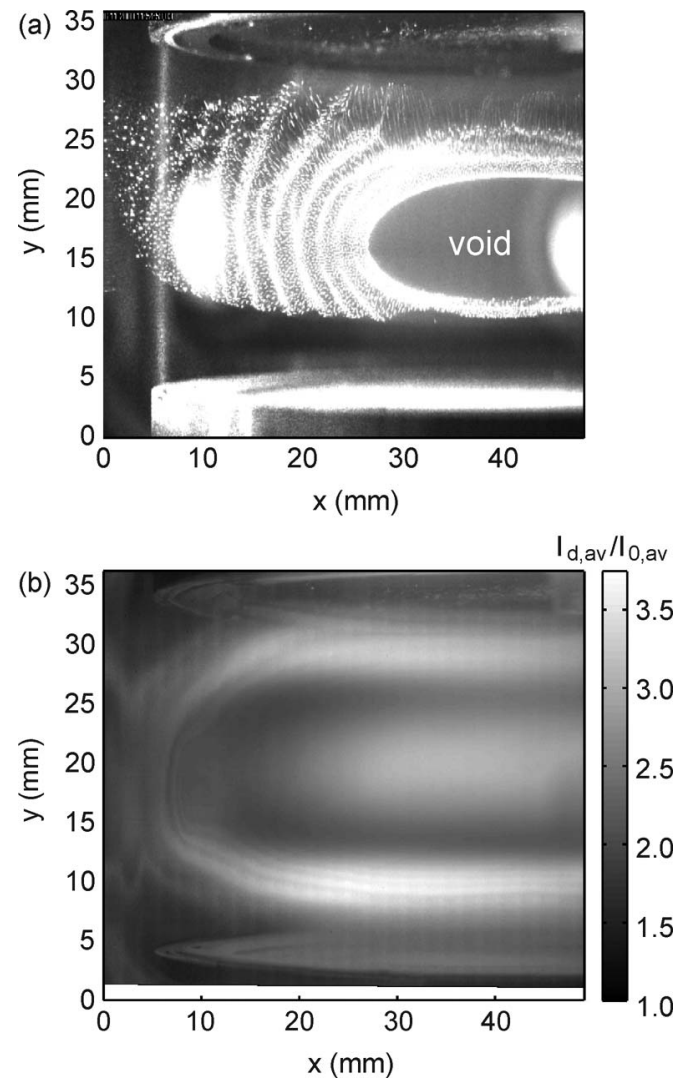

Fig. 2. (a) Snapshot of the dust cloud. Self-excited DDWs are propagating radially outward from the boundary of the dust-free "void" in the center of the discharge. (b) Glow presented as a ratio of the glow intensity for the dusty plasma with respect to the dust-free plasma, averaged over time.

An image of the plasma glow is presented in Fig. 2(b). To more clearly reveal the effects of dust, we have processed the glow image by computing, for each pixel, the intensity ratio $I_{d, \text { av }} / I_{0, \text { av }}$, where $I_{d, \text { av }}$ and $I_{0, \text { av }}$ are the glow intensity, averaged over many wave periods, for the plasma with and without dust, respectively. We see in Fig. 2(b) that the glow is enhanced everywhere in the plasma when dust is introduced, as indicated by a ratio $I_{d, \mathrm{av}} / I_{0, \mathrm{av}}>1$. The glow is most strongly increased, with maximum values $I_{d \text {,av }} / I_{0, \text { av }} \approx 3.5$, in the presheath regions in front of the electrodes and inside the void.

Fig. 3 reveals the effect of the DDW on the plasma glow. A snapshot of the spatially resolved fluctuation amplitude $\tilde{I}_{d}(x, y)$ of the dust density in units of the average local density $I_{d, \text { av }}(x, y)$ is shown in Fig. 3(a). One can see a wave pattern with a typical modulation depth of $\approx 30 \%$ of the average density value. The same diagram for the glow fluctuations is shown in Fig. 3(b). Obviously, the wave pattern inside the glow is not as pronounced as in the dust density. Only in the region above the void $(y=20-30 \mathrm{~mm})$, a significant modulation with a modulation depth of $\approx 5 \%$ of the average glow intensity is visible. To exclude possible crosstalk effects between the dust and the glow camera, we repeated the experiment after switching the illumination laser off. The glow fluctuations remained unchanged. Only after removing the dust from the discharge did the glow fluctuations disappear. Furthermore, we can exclude aliasing effects, since we compared the results 

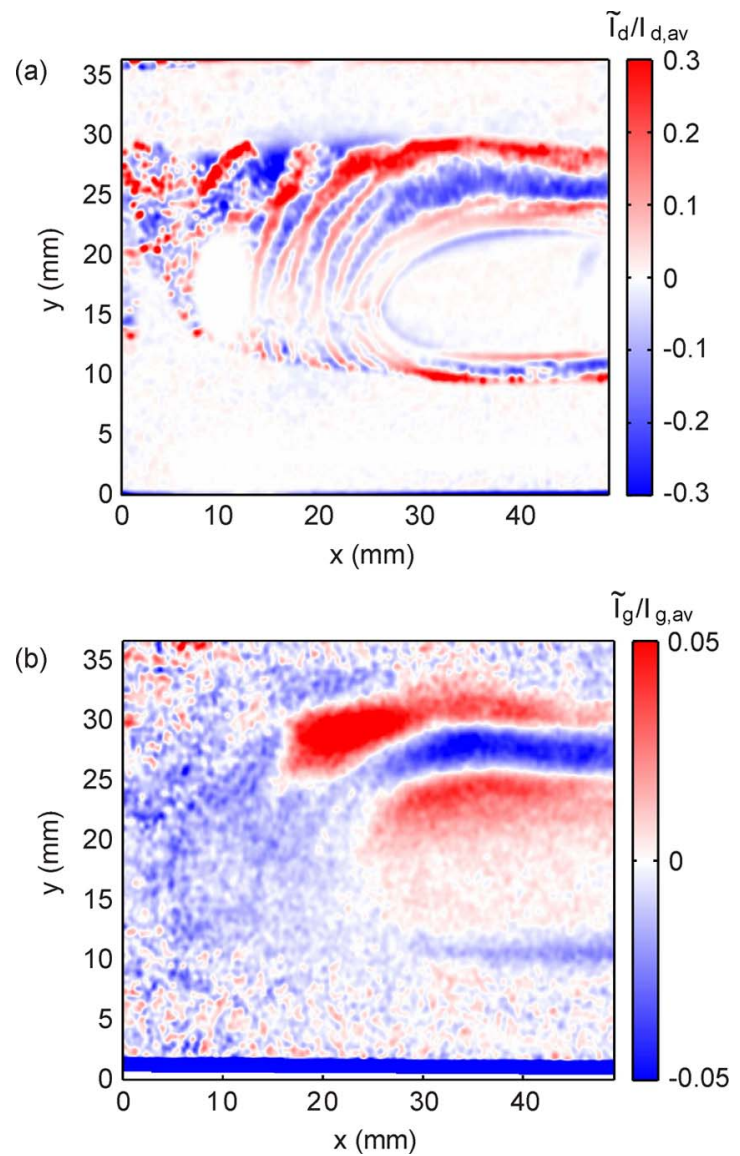

Fig. 3. Spatial amplitude map of (a) dust density fluctuations and (b) plasma glow fluctuations.

with measurements of a high-speed camera at a frame rate of 100 fps. The Fourier transforms of fluctuations in the dust density and the glow reveal a mainly monochromatic nature of the observed wave. Both power spectra, i.e., of the dust [Fig. 4(a)] and of the glow [Fig. 4(b)], were calculated at the same position $(x=42 \mathrm{~mm}, y=27 \mathrm{~mm})$ and show a dominant peak at the same frequency of $f=3.9 \mathrm{~Hz}$. As described earlier, we derive the phase of the wave by taking the argument of the complex Fourier coefficient corresponding to a frequency of $f=3.9 \mathrm{~Hz}$. The result is shown for the dust and the glow in Fig. 5(a) and (b) and is in excellent agreement with the fluctuation data shown in Fig. 3. The phase relation between the dust density and the glow, i.e., $\Delta \phi=\phi_{g}-\phi_{d}$, is shown in Fig. 6. Obviously, $\Delta \phi$ is almost constant in regions where significant wave motion was observable in both cameras, particularly above the void region. The average value inside the dashed region of interest is $\Delta \phi=0.66 \pi$, which corresponds to a phase difference of $118^{\circ}$, i.e., first comes the glow and afterward the dust density maximum.

\section{DisCUSSION AND CONCLUSION}

Our first main finding, i.e., an increased overall glow, is consistent with recent fluid simulations of a dusty plasma under microgravity conditions [11]. It was predicted that, in large dust clouds with a central void, the immense loss of plasma to the particles is compensated by an enhanced ionization due to
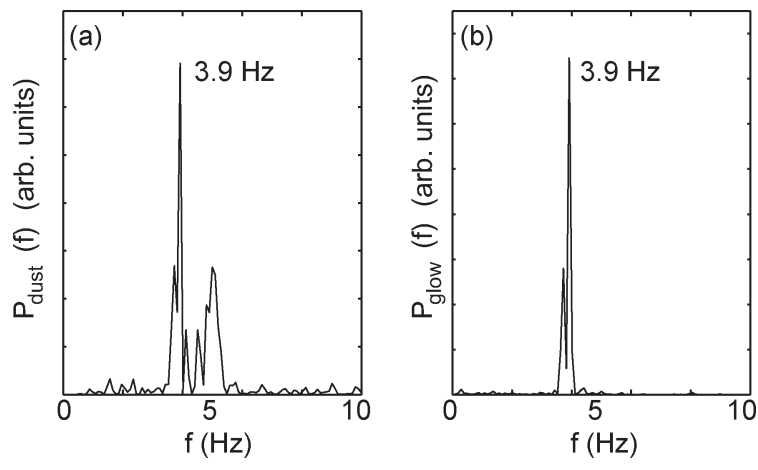

Fig. 4. Power spectra of the (a) dust density fluctuations and (b) glow fluctuations at the position $x=42 \mathrm{~mm}, y=27 \mathrm{~mm}$.
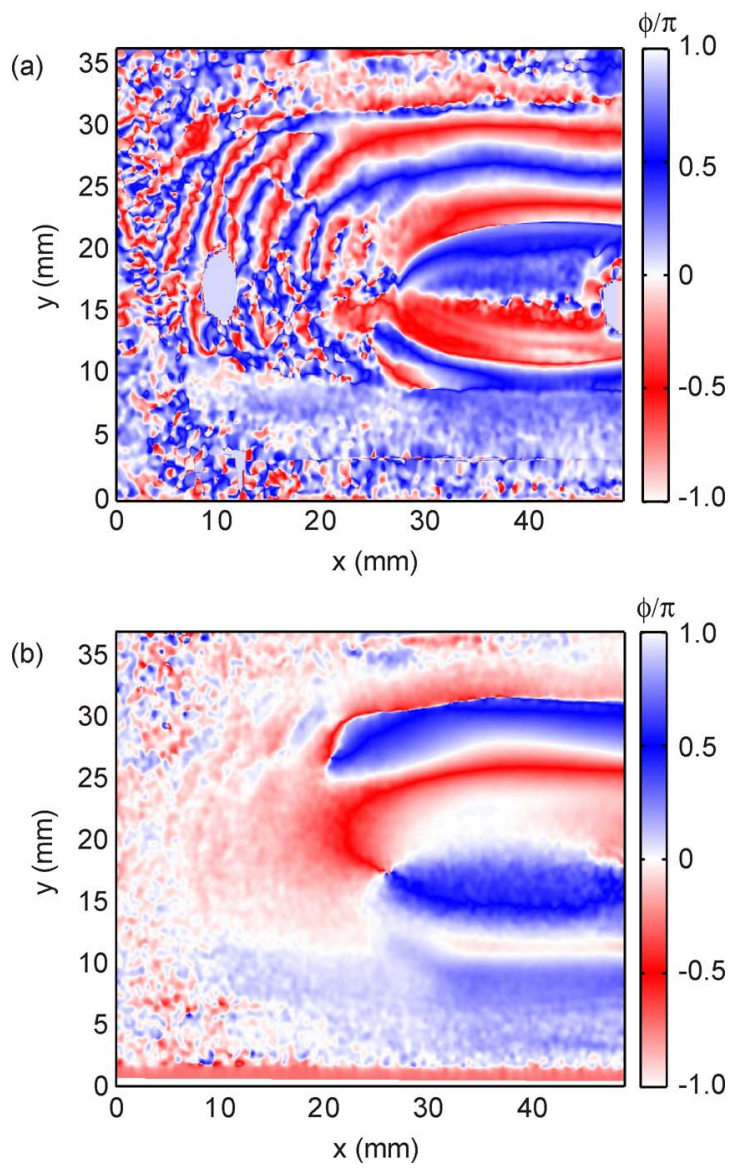

Fig. 5. Phase map of the (a) dust density fluctuations and (b) glow fluctuations derived from the Fourier transform. The phase is evaluated for the dominant wave frequency $f=3.9 \mathrm{~Hz}$.

increased electron temperature and density. This behavior can be described as a change of the impedance of the discharge due to the presence of the dust.

Our second main finding, that the plasma glow was significantly modulated by the DDW with a fixed-phase relation of $\Delta \phi=118^{\circ}$, reveals that the DDW has a more complex character than has previously been reported. The DDW is not merely a compressional modulation of the dust driven by an ion flow, but it also involves modulation of energetic electrons and electric field, as indicated by the glow. This suggests that it might also involve modulation of the ionization rate and dust charge as well. The modulation depth for the glow is not 


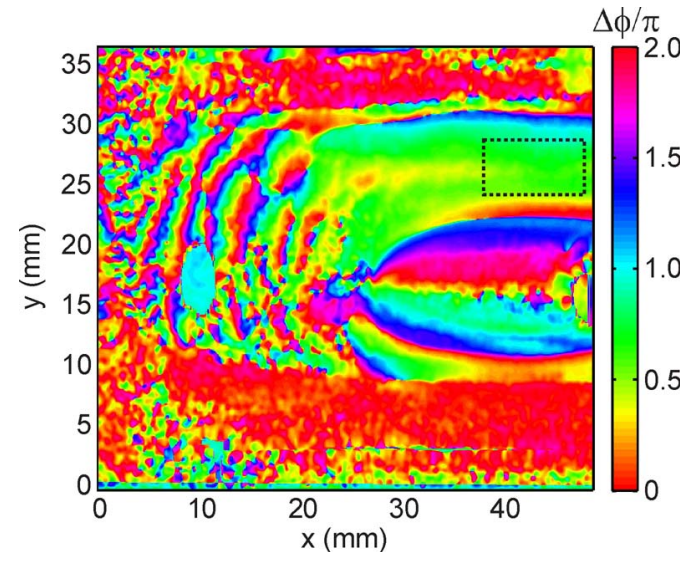

Fig. 6. Spatial map of the phase difference $\Delta \phi=\phi_{g}-\phi_{d}$ between the glow and the dust. The average value in the dashed region of interest is $\Delta \phi=0.66 \pi$, which corresponds to a phase difference of $118^{\circ}$.

as easily measured as for the dust. The dust is imaged as a cross section, because it is illuminated by a thin sheet of laser light. Images of the glow, on the other hand, are essentially chord-averaged over the entire volume of the chamber. This chord averaging will have the effect of reducing the apparent modulation, to an extent that is difficult to predict, depending on the 3-D shape of the wavefronts. The brightness of the dust was modulated by about $30 \%$, relative to its local average, whereas the glow images were modulated by only about $5 \%$ with respect to its local average. Aside from chord averaging of the glow, the calculation of these modulation fractions is also affected by the Gaussian filter, which slightly blurs the images and thereby leads to a smaller fluctuation amplitude.

For the interpretation of the phase relation between the dust density and plasma fluctuations, we rely on the following assumptions: First, we assume that the dust density is periodically disturbed by a Buneman-type instability. Second, the plasma inside the wave field is always in equilibrium, since the timescales required for the relaxation of fluctuations in the electron and, respectively, ion densities are by orders of magnitude smaller than the timescales of the DDWs. Hence, we conclude that the plasma instantaneously reacts on electric fields due to space charges as a consequence of inhomogeneities of the dust density. This causes a spatiotemporal modulation of the plasma potential, which is most strongly reduced at places with maximum dust density. Assuming, as a first approximation, a sinusoidal modulation of the dust density, we expect a maximum of the electric field inside the wave where the local dust density most strongly changes. This suggests a phase relation between the dust density and the electric field modulation of $90^{\circ}$. Consequently, when directly considering the plasma glow as an indicator for electric fields, one would expect a phase relation between the dust density and the plasma glow of $90^{\circ}$. At this time, it is unknown whether the deviation from the $90^{\circ}$ phase difference is entirely physical, or whether it could be partly due to artifacts from the Fourier analysis method arising from, e.g., strangely shaped or broken wavefronts.

Our results reveal that a complete understanding of DDWs is lacking. The observations of a modulated plasma glow indicate that the population of energetic electrons, and possibly the ionization rate and dust charge, is modulated as part of the wave, and in a self-consistent model, they cannot be assumed to be constant. A further complication is that (depending on the mean-free path for electron-neutral collisions), the physics might be nonlocal, in the sense that electrons might be accelerated by electric fields at one point and cause glow and enhanced ionization elsewhere.

\section{ACKNOWLEDGMENT}

The work at Iowa was supported by the National Aeronautics and Space Administration and the U.S. Department of Energy.

\section{REFERENCES}

[1] A. Barkan, R. L. Merlino, and N. D'Angelo, "Laboratory observation of the dust-acoustic wave mode," Phys. Plasmas, vol. 2, no. 10, pp. 35633565 , Oct. 1995.

[2] T. Trottenberg, D. Block, and A. Piel, "Dust confinement and dustacoustic waves in weakly magnetized anodic plasmas," Phys. Plasmas, vol. 13, no. 4, p. 042105, Apr. 2006.

[3] M. Schwabe, M. Rubin-Zuzic, S. Zhdanov, H. M. Thomas, and G. E. Morfill, "Highly resolved self-excited density waves in a complex plasma," Phys. Rev. Lett., vol. 99, no. 9, p. 095002, Aug. 2007.

[4] A. Piel, O. Arp, M. Klindworth, and A. Melzer, "Obliquely propagatingdust density waves," Phys. Rev. E, Stat. Phys. Plasmas Fluids Relat. Interdiscip. Top., vol. 77, no. 2, p. 026407, Feb. 2008.

[5] M. Rosenberg, "Ion-dust streaming instability in processing plasmas," J. Vac. Sci. Technol. A, Vac. Surf. Films, vol. 14, no. 2, pp. 631-633, Mar. 1996.

[6] N. N. Rao, P. K. Shukla, and M. Y. Yu, "Dust-acoustic waves in dusty plasmas,” Planet. Space Sci., vol. 38, no. 4, pp. 543-546, Apr. 1990.

[7] A. Piel, M. Klindworth, O. Arp, A. Melzer, and M. Wolter, "Obliquely propagating dust-density plasma waves in the presence of an ion beam," Phys. Rev. Lett., vol. 97, no. 20, p. 205 009, Nov. 2006.

[8] A. Piel, M. Klindworth, O. Arp, A. Melzer, and M. Wolter, "Erratum: Obliquely propagating dust-density plasma waves in the presence of an ion beam," Phys. Rev. Lett., vol. 99, no. 20, p. 209903, Nov. 2007.

[9] G. Praburam and J. A. Goree, "Experimental observation of very lowfrequency macroscopic modes in a dusty plasma," Phys. Plasmas, vol. 3, no. 4, pp. 1212-1219, Apr. 1996.

[10] D. Samsonov and J. A. Goree, "Instabilities in a dusty plasma with ion drag and ionization," Phys. Rev. E, Stat. Phys. Plasmas Fluids Relat. Interdiscip. Top., vol. 59, no. 1, pp. 1047-1058, Jan. 1999.

[11] V. Land and W. J. Goedheer, "The plasma inside a dust free void: Hotter, denser, or both?" New J. Phys., vol. 9, no. 8, p. 246, 2007.

Oliver Arp received the Ph.D. degree in physics from Christian-AlbrechtsUniversität zu Kiel (CAU), Kiel, Germany, in 2006.

He is currently a Postdoctoral Research Fellow with CAU and the coordinator of the microgravity experiment.

David Caliebe received the Diploma degree in physics in 2008 from ChristiansAlbrechts-Universität zu Kiel (CAU), Kiel, Germany, where he is currently working toward the Ph.D. degree under the supervision of Prof. Piel.

He has participated on several parabolic flight campaigns. His interests include transport phenomena in dusty plasmas under microgravity.

Kristoffer Ole Menzel received the Diploma degree in physics in 2008 from Christians-Albrechts-Universität zu Kiel (CAU), Kiel, Germany, where he is currently working toward the Ph.D. degree under the supervision of Prof. Piel.

He has participated on several parabolic flight campaigns. His interests include the dynamics of density waves in dusty plasmas under microgravity. 
Alexander Piel was born in 1950. He received the Ph.D. and Habilitation degrees from Ruhr-University Bochum, Bochum, Germany, in 1977 and 1986, respectively.

Since 1989, he has been a Full Professor with Christian-AlbrechtsUniversität zu Kiel, Kiel, Germany. His research interests cover dusty plasmas, plasma waves, and ionospheric research.

Dr. Piel is a Fellow of the American Physical Society.
John A. Goree (M'92) received the Ph.D. degree from Princeton University, Princeton, NJ, in 1985. He is currently with the Department of Physics and Astronomy, University of Iowa, Iowa City. 